\title{
Nuclear quantum state engineering in ion channeling regime
}

\author{
Vesna Berec ${ }^{1,2 a}$ \\ ${ }^{1}$ Institute of Nuclear Sciences, P. O. Box 522, Belgrade, Serbia \\ ${ }^{2}$ University of Belgrade, Serbia
}

\begin{abstract}
A key challenge in quantum state engineering is to identify coherent quantum mechanical systems that can be precisely manipulated and scaled, but at the same time to allow decoupling from unwanted interactions. Such systems, once realized, would represent an efficient tool for characterization of quantum behavior reflected in the properties of matter with prerequisites for meeting dissipation constraints imposed in the nuclear physics as well in the quantum information theory. Using the pure ${ }^{29} \mathrm{Si}$ nanocrystal system we present a novel high resolution method for initialization of single electron polarized spin interaction and control of nuclear spin qubits. The presented study fuses field of particle channeling in $\mathrm{MeV}$ energy regime with quantum state engineering utilized via entanglement as an essential quantum property. Its aim is to bring focus on new theoretical proposals testing the quantum mechanical models for systems producible at particle accelerator facilities.
\end{abstract}

\section{Introduction}

Discovering of powerful quantum algorithms (Shor [1]; Grover [2]) has initialized development of quantum information concepts and facilitated novel pathways in theory and application of complex systems including the quest for efficient resources of quantum entanglement as a specific correlation, establishing new demands for ultimate control and manipulation over subatomic systems. Following these strategies, in this work we demonstrate theoretically quantum control of subatomic particle states using a particular axial mode of channeling founded by Robinson and Oen [3] which allows high resolution localization of subatomic particles induced by enhancement of confining potential inside ultra thin crystal targets [4]. This effect is established when the particle trajectory is confined to one axial channel during its transmission through a very thin perfect crystalline targets (due to small percentage of energy losses) for very small angles of incidence and negligible beam divergence.

It is considered that the nuclei of silicone atoms, which form atomic strings defining the crystal channel, represent the nuclear spin qubits. Thus, the insertion of a foreign atom in desired location inside crystal target (whose thickness corresponds to one atomic layer) can be performed in order to establish ancillary qubit. Our goal is to minimize interaction of quantum system with environment and to prevent decay of information (to reduce amount of decoherence). In order to achieve that computation must be completed before the information has significantly decayed.

${ }^{\text {a }}$ Corresponding author: bervesn@gmail.com 
In order to address issues of collective effects and quantum correlations resulting from the interplay between resonance and specific coherent effects in channeling mode, we demonstrate a simple simulation model based on the quantum game protocol [5] that includes and explain the quantitative feedback relation of the nuclear qubit quantum resonance states, which are achieved vs control information states coming from sharply collimated particle beam.

In the next section we start from the Hamiltonian of the Lindblad form where the master equation is applicable to the quantum two player game, as

$$
\frac{d \rho}{d t}=-i[H(t), \rho]+\sum_{j}\left(\gamma_{j} L_{j} \rho L_{j}^{\dagger}-\frac{1}{2}\left\{L_{j}^{\dagger} L_{j}, \rho\right\}\right),
$$

where $H$ denotes the system Hamiltonian, $L_{j}$ are the Lindblad operators, representing the coherence detriment in the system and $\rho$ is the quantum state of the system. The system dynamics is expressed using the concepts of quantum systems control. Moreover, we implement this approach to the case in which the system is governed by an internal Hamiltonian that corresponds to anisotropic hyperfine interaction which occurs during the transmission channeling mode [6].

\section{Analytical and numerical protocol}

Block diagonal system Hamiltonian which is incorporated in the above Linbland equation is:

$$
H=\omega_{e} \widehat{S}_{z}+\omega_{n} \hat{I}_{z}+2 \pi A_{z z} \widehat{S}_{z} \hat{I}_{z}+2 \pi A_{\perp} \widehat{S}_{z} \widehat{I}_{x}
$$

where $\widehat{S}$ and $\widehat{I}$ refer to components of electron and spin operators, $\omega_{e}=\omega_{s}-\omega_{\alpha, \beta}$ and $\omega_{n}$ associate electron and nuclear frequencies, respectively, and $\omega_{\alpha, \beta}$ are resonance transition frequencies induced by the channeled particle beam. The last term denotes the anisotropic hyperfine interaction: $A_{z x} \widehat{S}_{z} \hat{I}_{x}+A_{z y} \widehat{S}_{z} \hat{I}_{y}$, where the anisotropic hyperfine coupling tensor is $A_{\perp}=\sqrt{A_{z x}{ }^{2}+A_{z y}{ }^{2}}$. As a next step, we consider a preparation device which can produce a quantum system considering inputs states: $|00\rangle,|01\rangle,|10\rangle,|11\rangle$. Using a 2 bit SWAP operation (quantum gate):

$$
S W A P=\left(\begin{array}{llll}
1 & 0 & 0 & 0 \\
0 & 0 & 1 & 0 \\
0 & 1 & 0 & 0 \\
0 & 0 & 0 & 1
\end{array}\right),
$$

the four inputs states are mapped, respectively, into: $|01\rangle \rightarrow|1\rangle,|00\rangle \rightarrow|2\rangle,|10\rangle \rightarrow|3\rangle,|11\rangle \rightarrow$ 14) state. Consequently, the $S W A P$ operation is used in this initial stage to describe polarization transfer from the channeled beam particle states to electron-nuclear spin subsystem in silicone target:

$$
\begin{aligned}
& U_{p o l}=U_{D}^{\dagger} S W A P U_{D} \\
& U_{D}=e^{-i\left(\theta_{\uparrow} E_{+}^{e} I_{x y}+\theta_{\downarrow} E_{-x y}^{e} I_{x y}\right)},
\end{aligned}
$$

where $E_{ \pm}^{e}$ are the polarization operators for electron spin, and $\theta_{\uparrow}$ and $\theta_{\downarrow}$ are the corresponding quantization axis angles. 
Unitary transformation is applied over individual qubits by selective pulses with specific rotation angle equaled to corresponding percentages (0-15\%) of the critical angle for axial channeling [6, 7], see figure 1. Thickness of the silicone target is one atomic layer. Channeled beam sequence, for the spin qubits in the $x-y$ direction of the rotation frame, initializes unitary transformation coinciding with expression for Pauli matrices as:

$$
P_{j, k}(\vartheta)=I_{4} \cos \left(\frac{\vartheta}{2}\right)-i \sigma_{x, y} \sin \left(\frac{\vartheta}{2}\right)
$$
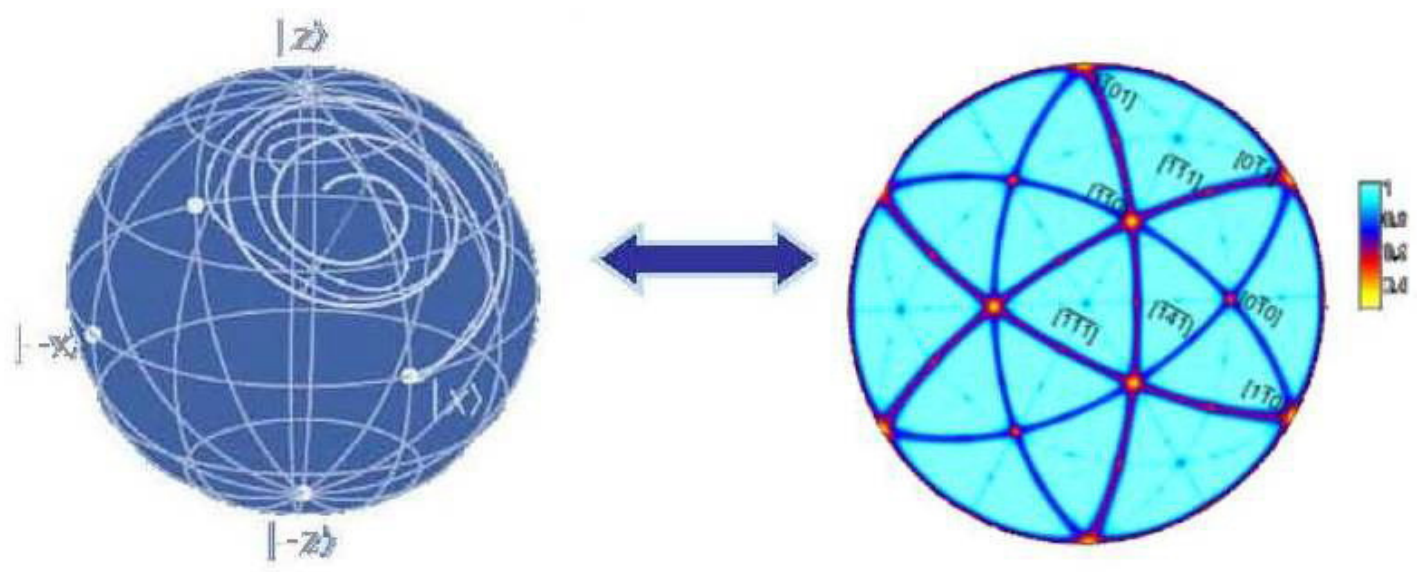

Figure 1. Scheme of the trajectory executed by the nuclear spin initially oriented along the $\mathbf{z}$ axis when the channeled beam sequence is applied; the desired rotation is implemented by switching the electron spin transition frequencies $\omega_{i}(i=1, \ldots, 4)$ Eq. (13), between electron spin manifolds: $E_{+}^{e}=1 / 2 \mathbb{I}+S_{z}, E_{-}^{e}=1 / 2 \mathbb{I}-S_{z}$. Right: corresponding trajectories executed via channeled particle beam under specified condition of the critical angle for channeling [6, 7], for $\varphi \leq 15 \% \psi_{c}$.

After applying the entangling operation:

$$
J_{P_{j k} D}\left(S_{x}\right)|00\rangle=(-1)^{S_{x} / 2 \pi}(|00\rangle+|11\rangle) / 2+(-1)^{-S_{x} / 2 \pi}(|01\rangle+|10\rangle) / 2,
$$

and using the operator $D=U(\pi, 0)=|0\rangle\langle 1|-| 1\rangle\langle 0|$, the set of electron-nuclear subsystems becomes coupled as following:

$$
\begin{aligned}
& \left\langle 1\left|S_{x}\right| 4\right\rangle=\cos \left(\theta_{\uparrow}-\theta_{\downarrow}\right), \\
& \left\langle 1\left|S_{x}\right| 3\right\rangle=\sin \left(\theta_{\uparrow}-\theta_{\downarrow}\right), \\
& \left\langle 2\left|S_{x}\right| 3\right\rangle=\cos \left(\theta_{\downarrow}-\theta_{\uparrow}\right), \\
& \left\langle 2\left|S_{x}\right| 4\right\rangle=\sin \left(\theta_{\downarrow}-\theta_{\uparrow}\right),
\end{aligned}
$$

where: 


$$
\begin{aligned}
& |1\rangle=\left|\uparrow \alpha_{0}\right\rangle=|\uparrow\rangle \otimes\left(\sin \theta_{\uparrow}|\uparrow\rangle+\cos \theta_{\uparrow}|\downarrow\rangle\right), \\
& |2\rangle=\left|\uparrow \alpha_{1}\right\rangle=|\uparrow\rangle \otimes\left(\cos \theta_{\uparrow}|\uparrow\rangle-\sin \theta_{\uparrow}|\downarrow\rangle\right), \\
& |3\rangle=\left|\downarrow \beta_{1}\right\rangle=|\downarrow\rangle \otimes\left(\cos \theta_{\downarrow}|\uparrow\rangle-\sin \theta_{\downarrow}|\downarrow\rangle\right), \\
& |4\rangle=\left|\downarrow \beta_{0}\right\rangle=|\downarrow\rangle \otimes\left(\sin \theta_{\downarrow}|\uparrow\rangle+\cos \theta_{\downarrow}|\downarrow\rangle\right) ; \quad(|\uparrow\rangle=|0\rangle,|\downarrow\rangle=|1\rangle) .
\end{aligned}
$$

In the former Eq. (7): $\alpha_{0}, \alpha_{1}, \beta_{1}, \beta_{2}$ are the mixing coefficients of the resonance transition frequencies induced during the transmission channeling mode over hyperfine coupling tensor components $A_{z z}$ and $A_{\perp}$ :

$$
\begin{aligned}
& \omega_{\alpha_{0}, \alpha_{1}}=\Omega_{ \pm}^{12}=\left(\left(m_{s} A_{z z}+\omega_{n}\right)^{2} \pm\left(m_{s} A_{\perp}\right)^{2}\right)^{1 / 2} \\
& \omega_{\beta_{0}, \beta_{1}}=\Omega_{ \pm}^{34}=\left(\left(m_{s} A_{z z}-\omega_{n}\right)^{2} \pm\left(m_{s} A_{\perp}\right)^{2}\right)^{1 / 2} .
\end{aligned}
$$

$S_{x}$ operator originates from the interaction Hamiltonian: $H_{1}=\frac{\beta_{e}}{\hbar} g_{e} \vec{B}_{1}(t) \cdot \vec{S}$, i.e., from the interaction established during the channeling of a particle beam.

Relations (6) and (7) address the anisotropic hyperfine coupling tensor, $A_{\perp}$, via quantization axis angles, $\theta_{\uparrow}$ and $\theta_{\downarrow}$, to a $z$ component of the contact hyperfine interaction tensor (see figure 2) as:

$$
\begin{aligned}
& \theta_{\uparrow}=\frac{1}{2} \tan ^{-1}\left(-\frac{A_{\perp}}{A_{z z}-\frac{\omega_{I}}{\pi}}\right), \\
& \theta_{\downarrow}=\frac{1}{2} \tan ^{-1}\left(-\frac{A_{\perp}}{A_{z z}+\frac{\omega_{I}}{\pi}}\right) .
\end{aligned}
$$

The case when $\theta_{\uparrow}=\theta_{\downarrow}$, see Eq. (6), excludes the electron spin transition frequencies $\omega_{13}, \omega_{24}$ and forbids the transitions between the states $|1\rangle \leftrightarrow|3\rangle$ and the states $|2\rangle \leftrightarrow|4\rangle$.

For the case when $\theta_{\uparrow} \neq \theta_{\downarrow}$, activated transition frequencies, $\omega_{13}$ and $\omega_{24}$, see figure 2 right, are responsible for establishing quantum correlations in electron spin manifold due the anisotropic hyperfine couplings between electron and nuclear spin subsystems.

The setting of coupled e-n spin transition frequencies, see figure 2, which are responsible for establishment of quantum correlation between nuclear spins in electron spin manifold, completely coincides with the setting of two parameter quantum strategy defined by Eisert et. al. [5], which is based on the following set of Eqs. $(11,12)$ :

$$
S=\{M(\vartheta, \phi): \vartheta \in[0, \pi], \phi \in[0, \pi / 2]\},
$$




$$
M(\vartheta, \phi)=\left[\begin{array}{cc}
e^{i \phi} \cos (\vartheta / 2) & i \sin (\vartheta / 2) \\
i \sin (\vartheta / 2) & e^{-i \phi} \cos (\vartheta / 2)
\end{array}\right]
$$

as:

$$
\omega_{1}=\left[\begin{array}{cc}
e^{i \phi_{1}} \cos \left(\vartheta_{1} / 2\right) & i \sin \left(\vartheta_{1} / 2\right) \\
i \sin \left(\vartheta_{1} / 2\right) & e^{-i \phi_{1}} \cos \left(\vartheta_{1} / 2\right)
\end{array}\right], \ldots \omega_{4}=\left[\begin{array}{cc}
e^{i \phi_{4}} \cos \left(\vartheta_{4} / 2\right) & i \sin \left(\vartheta_{4} / 2\right) \\
i \sin \left(\vartheta_{4} / 2\right) & e^{-i \phi_{4}} \cos \left(\vartheta_{4} / 2\right)
\end{array}\right],
$$

where the dimension of coupling frequencies: $\omega_{1} \otimes \omega_{1} \otimes \cdots \omega_{4}$ is $2^{4} \times 2^{4}$ and specific coupling frequencies are denoted as following:

$$
\begin{aligned}
& \omega_{23}=\omega_{e}+1 / 2\left(\omega_{12}-\omega_{34}\right), \\
& \omega_{14}=\omega_{e}-1 / 2\left(\omega_{12}-\omega_{34}\right), \\
& \omega_{13}=\omega_{e}+1 / 2\left(\omega_{12}+\omega_{34}\right), \\
& \omega_{24}=\omega_{e}-1 / 2\left(\omega_{12}+\omega_{34}\right) .
\end{aligned}
$$
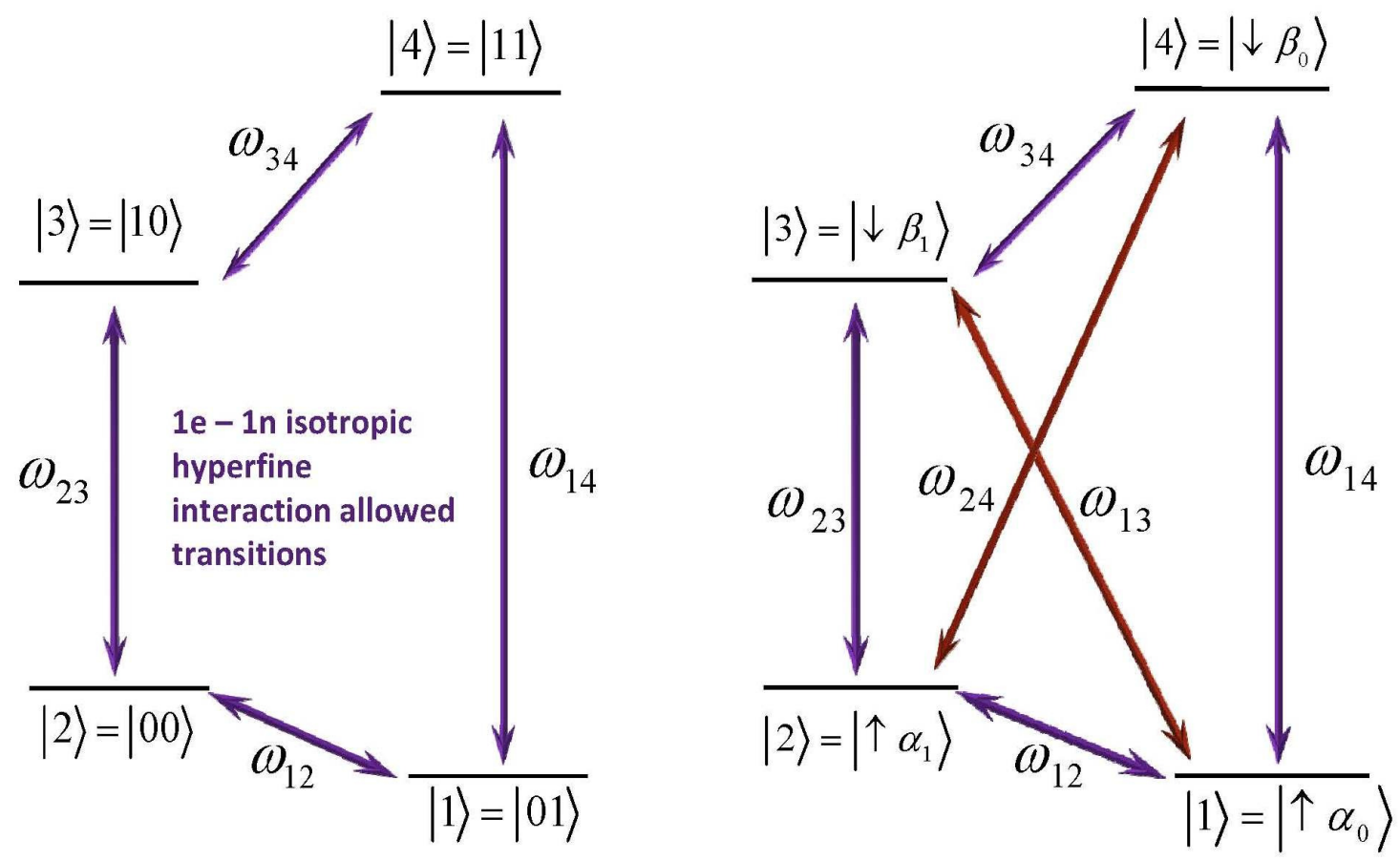

Figure 2. Scheme of quantum correlations established between the hyperfine coupled electron $(S=1 / 2)$ and nuclear $(I=1 / 2)$ spin in two two-level system. Left: activated quantum transitions (frequencies) via isotropic hyperfine interaction. Right: activated quantum transitions (frequencies) via anisotropic hyperfine interaction.

Note: we can achieve maximum entanglement power of $\frac{2}{9}$ for the coupling frequencies settings also, using the matrix, M, which maps the computational basis into the "magic" basis, via procedure: MUM, where $\mathrm{U}$ is purely real unitary matrix: 


$$
\begin{aligned}
\mathrm{M} & =\left|M_{1}\right\rangle\left\langle 00|+| M_{2}\right\rangle\left\langle 01|+| M_{3}\right\rangle\left\langle 10|+| M_{4}\right\rangle\langle 11| \\
& =\left|M_{1}\right\rangle\left\langle 2|+| M_{2}\right\rangle\left\langle 1|+| M_{3}\right\rangle\left\langle 3|+| M_{4}\right\rangle\langle 4| \\
& =\frac{1}{\sqrt{2}}\left(\begin{array}{cccc}
1 & i & 0 & 0 \\
0 & 0 & i & 1 \\
0 & 0 & i & -1 \\
1 & -i & 0 & 0
\end{array}\right)
\end{aligned}
$$

Former sequence is achieved applying the gates:

$$
\begin{aligned}
& S=P_{h}\left(\frac{\pi}{4}\right) \cdot R_{z}\left(\frac{\pi}{2}\right) \text {, and } \mathrm{H}=Z \cdot R_{y}\left(-\frac{\pi}{2}\right) \text {, where } P_{h}(\gamma)=e^{i \gamma}\left(\begin{array}{ll}
1 & 0 \\
0 & 1
\end{array}\right) ; \\
& \mathrm{R}_{y}(\alpha)=\exp (-i \alpha Y / 2)=\left(\begin{array}{cc}
\cos (\alpha / 2) & -\sin (\alpha / 2) \\
\sin (\alpha / 2) & \cos (\alpha / 2)
\end{array}\right) ; \text { and } \mathrm{R}_{Z}(\alpha)=\exp (-i \alpha Z / 2)=\left(\begin{array}{cc}
e^{-i \alpha / 2} & 0 \\
0 & e^{i \alpha / 2}
\end{array}\right) .
\end{aligned}
$$

Correspondingly, the transition frequencies $\omega_{23}, \omega_{14}$ and $\omega_{13}, \omega_{24}$ are equal when the phase shift gate is set to $P_{h}(\gamma)=e^{i \pi / 4}\left(\begin{array}{ll}1 & 0 \\ 0 & 1\end{array}\right)$ coinciding to the case when $\left|\theta_{\downarrow}-\theta_{\uparrow}\right|=\pi / 4$.

\section{Conclusion}

We have demonstrated analytical and numerical procedure for quantum control of correlations in two level electron-nuclear $1 / 2$ spin system. The obtained values of the transition frequencies are represented using the strategies of Eisert protocol, based on the quantum two player game. A solution concept of the established quantum protocols represent a function that captures specific amount of quantum correlation and associate to each qubit a subset of transition frequencies which are responsible for quantum control.

\section{Acknowledgment}

Author is indebted to Choo Hiap $\mathrm{OH}$ and Peter Minkowski for fruitful discussions. In particular, author would like to thank to 'ICNFP2014' on the financial support for this contribution.

\section{References}

1. P. W. Shor, SIAM J. Comput. 26 (5),1484-1509 (1997)

2. L. K. Grover, American Journal of Physics, 69 (7), 769-777 (2001)

3. M. T. Robinson, and O. S. Oen, Phys. Rev. 132, 2385 (1963)

4. V. Berec, Laser and Particle Beams (2014) DOI: 10.1017/S0263034614000482 Cambridge University Press

5. J. Eisert, M. Wilkins, M. Lewenstein, Phys. Rev. Lett. 83, 3077 (1999)

6. V. Berec, PLoS ONE 7(9), e45254 (2012) DOI: 10.1371/journal.pone.0045254

7. J. Lindhard, K. Dan. Vidensk. Selsk., Mat.-Fys. Medd. 34 (14), 1 (1965). 